\title{
A Case of 'Sweet' Hydrothorax in a Patient on Peritoneal Dialysis
}

\author{
Nejc Piko ${ }^{1}$, Martin Hren ${ }^{1}$, Sebastjan Bevc ${ }^{1,2}$, Robert Ekart ${ }^{2,3}$ \\ ${ }^{1}$ Department of Nephrology, Clinic for Internal Medicine, University Medical Centre Maribor, Maribor, Slovenia \\ ${ }^{2}$ Medical Faculty Maribor, University of Maribor, Maribor, Slovenia \\ ${ }^{3}$ Department of Dialysis, Clinic for Internal Medicine, University Medical Centre Maribor, Maribor, Slovenia
}

\section{Doi: 10.12890/2019_001060 - European Journal of Case Reports in Internal Medicine - 다 EFIM 2019}

Received: 28/01/2018

Accepted: 29/01/2018

Published: $27 / 02 / 2019$

How to cite this article: Piko N, Hren M, Bevc S, Ekart R. A case of "sweet" hydrothorax in a patient on peritoneal dialysis. EJCRIM 2019;6: doi:10.12890/2019_001060.

Conflicts of Interests: The Authors declare that there are no competing interests.

This article is licensed under a Commons Attribution Non-Commercial 4.0 License

\section{HOSPITAL GRAND ROUNDS}

UKC Maribor, by Editorial Board Member Radovan Hojs

University Medical Centre (UKC) Maribor, Slovenia, provides secondary and tertiary healthcare services to patients from Maribor and northeastern part of Slovenia. It has status of teaching hospital and it is learning base for medical students. The origin of UKC Maribor reaches back to the Maribor city hospital that has been treating patients since 14th century and constantly developing since its official establishment in 1799. Today UKC Maribor employs 3400 staff members, of them approx. 600 medical doctors.

Part of UKC Maribor is Clinic for Internal medicine that includes departments of Cardiology and Angiology, Gastroenterology, Endocrinology and Diabetology, Nephrology, Dialysis, Rheumatology, Haematology and Hematologic Oncology, Pulmonary Diseases, Nuclear Medicine, Internal Intensive Medicine and Emergency Medical Unit.

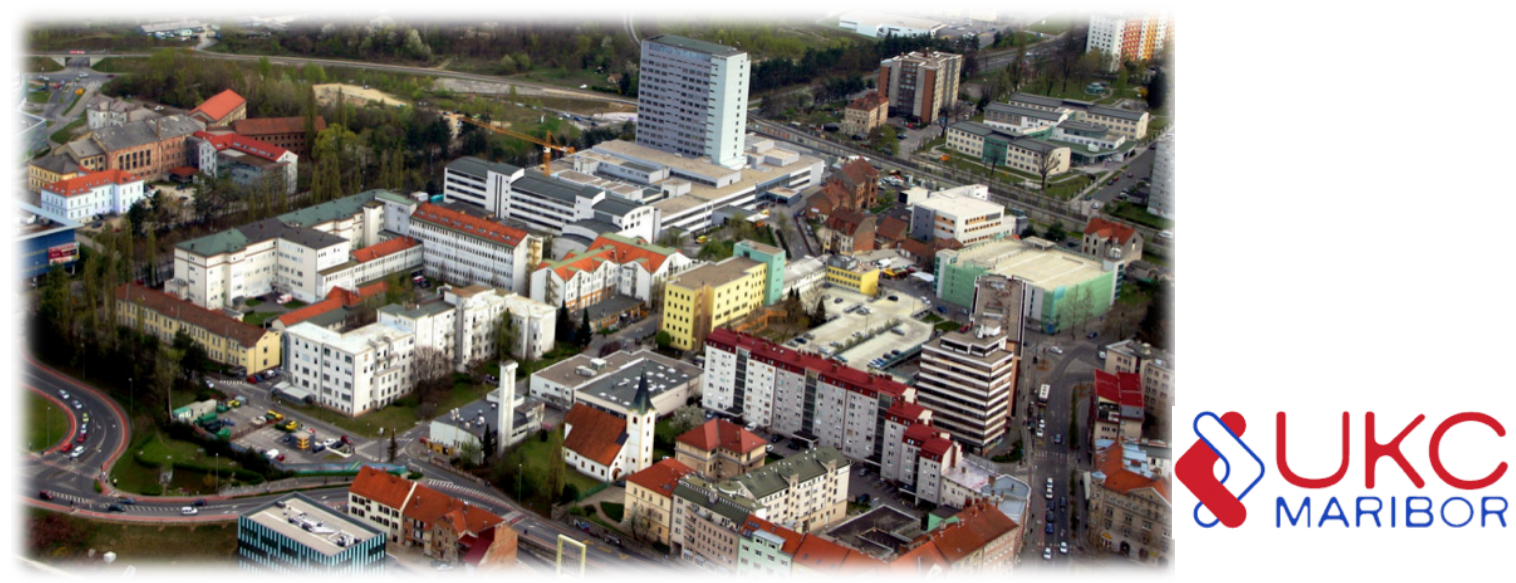

University Medical Centre Maribor 


\section{ABSTRACT}

Non-infectious complications are an important cause of peritoneal dialysis failure. Increased intra-abdominal pressure resulting from dialysate inflow into the peritoneal cavity can cause leaks, including hydrothorax due to pleuroperitoneal communication. The authors describe a patient on peritoneal dialysis with a newly discovered pleural effusion with a high glucose level. The patient was treated conservatively with peritoneal dialysis cessation and switched to haemodialysis with complete resolution of the pleural effusion. After 5 weeks, the patient successfully restarted peritoneal dialysis without recurrence of the hydrothorax.

\section{LEARNING POINTS}

- Pleural effusion in a patient on peritoneal dialysis can be caused by leakage of dialysate through pleuroperitoneal communication.

- Pleural effusion as a result of dialysate leak is rich in glucose.

- If conservative treatment with temporary peritoneal dialysis cessation is unsuccessful, any pleuroperitoneal communication should be surgically repaired.

\section{KEYWORDS}

Peritoneal dialysis, pleuroperitoneal communication, pleural effusion

\section{INTRODUCTION}

Peritoneal dialysis (PD), haemodialysis (HD) and kidney transplantation are offered to patients with end-stage renal disease (ESRD). Kidney transplantation is the gold standard, offering the longest patient survival. The differences in prognosis between PD and HD patients are not as well known, with some studies showing improved survival of patients on PD, especially in the first few years of renal replacement therapy ${ }^{[1]}$. Due to its efficacy, high patient satisfaction and lower costs compared with HD, PD remains an important dialysis option ${ }^{[1]}$. In the past few years, the rates of infectious complications, especially peritonitis, have been reduced. However, this achievement has not been matched by improved management of non-infectious complications of $\mathrm{PD}^{[2]}$.

\section{CASE DESCRIPTION}

A 50-year-old man with known ESRD due to arterial hypertension and treated with continuous ambulatory PD for the previous 2 years, presented to the emergency department with dyspnoea. He had noticed reduced dialysate drainage during the previous week. Dyspnoea had started 3 days earlier and had progressively worsened. He had no history of cough, fever, chills, or pain in the abdomen or chest. At admission, he was afebrile and tachydyspnoic, his blood pressure was 120/85 mm Hg, heart rate was 120/minute and his oxygen saturation was $95 \%$. Auscultation of the right lung showed diminished breath sounds, while breathing on the left side was normal. He did not have oedema in his legs. The position of the PD catheter was normal, there was no infection or discharge at the exit site, and the patient had no signs of peritonitis.

An initial laboratory investigation showed mild normocytic anaemia, elevated urea and creatinine levels due to ESRD, normal electrolytes, a normal $\mathrm{pH}$ and bicarbonate level, low inflammatory parameters, normal liver function, and a normal albumin level. Tumour markers, immunological parameters and thyroid hormone levels were normal. Chest x-ray showed a right-sided pleural effusion.

Diagnostic and therapeutic pleural drainage was performed. A total of 7 litres of pleural fluid was gradually drained over 4 days. Biochemical analysis of the fluid showed it was a transudate with an unusually high level of glucose ( $15.3 \mathrm{mmol} / \mathrm{l} \mathrm{vs} 5.4 \mathrm{mmol} / \mathrm{l}$ in blood), while pleural fluid protein and lactate dehydrogenase (LDH) were normal. Microbiological analysis of the pleural fluid was negative.

Due to reduced drainage volumes, treatment with PD was temporarily stopped and the patient was started on HD. After a few days, a control chest $\mathrm{x}$-ray showed a significantly smaller right-sided pleural effusion. The patient felt much better and no longer had difficulty breathing. After 5 weeks on HD, the patient restarted PD, initially with a smaller inflow volume of dialysate. He had no recurrence of pleural effusion and is still treated with continuous ambulatory PD after 1.5 years.

\section{DISCUSSION}

The most frequent and important complication of PD is infection, which may result in catheter loss and discontinuation of PD. However, some evidence suggests that transfer to HD for these reasons may be decreasing ${ }^{[2]}$. Non-infectious complications of PD are also an important cause of PD technique failure and are most commonly due to increased intra-abdominal pressure resulting from the inflow of dialysate into the peritoneal cavity ${ }^{[2,3]}$.

The development of pleural effusion in a patient on PD is rare, being seen in 1.6-10\% of patients on PD, and may be due to several different reasons, most commonly volume overload, heart failure or local pleural processes. 
The described patient had right-sided pleural effusion without peripheral oedema, which suggested a patent pleuroperitoneal communication ${ }^{[4]}$.

Most pleural effusions present in the first year after PD initiation, with only $20 \%$ occurring after 1 year. In this case, the patient presented with hydrothorax after 2 years on PD. In the literature, hydrothorax has been found in patients up to 8 years after initiation of PD ${ }^{[5]}$.

The presence of a pleuroperitoneal communication should be suspected in a dyspnoic non-oedematous PD patient with a history of reduced dialysate drainage and failure of ultrafiltration. The diagnosis is based on confirmation that the fluid in the pleural space is indeed dialysate. Many different treatments have been described in the literature, but the most clinically simple and useful method is thoracocentesis with analysis of the pleural fluid. A transudate with an unusually high glucose level, as seen in our patient, supports the diagnosis of a patent pleuroperitoneal communication ${ }^{[5]}$.

Treatment consists of draining the peritoneal cavity and avoiding overnight dwells; more invasive procedures, such as pleurodesis or repair, are sometimes needed. A temporary or permanent switch to HD is required. The described patient was temporarily switched to HD and restarted on PD after 5 weeks, but with smaller initial inflow volumes. He had no recurrence of pleural effusion. However, studies show that most patients with pleuroperitoneal leaks ultimately require transfer to HD. Close monitoring is therefore needed in these patients ${ }^{[5,6]}$.

\section{REFERENCES}

1. Sinnakirouchenan R, Holley JL. Peritoneal dialysis versus hemodialysis: risks, benefits, and access issues. Adv Chronic Kidney Dis 2011;18:428-432.

2. McCormick BB, Bargman JM. Noninfectious complications of peritoneal dialysis: implications for patient and technique survival. J Am Soc Nephrol 2007;18:3023-3025.

3. Teitelbaum I, Burkart J. Peritoneal dialysis. Am J Kidney Dis 2003;42:1082-1096.

4. Bae EH, Kim CS, Choi JS, Kim SW. Pleural effusion in a peritoneal dialysis patient. Chonnam Med J 2011;47:43-44.

5. Lew SQ. Hydrothorax: pleural effusion associated with peritoneal dialysis. Perit Dial Int 2010;30:13-18.

6. Szeto CC, Chow KM. Pathogenesis and management of hydrothorax complicating peritoneal dialysis. Curr Opin Pulm Med 2004;10:315-319. 